\title{
. \\ ATITUDE EMPREENDEDORA: VALIDAÇÃO DE UM INSTRUMENTO DE MEDIDA COM BASE NO MODELO DE RESPOSTA GRADUAL DA TEORIA DA RESPOSTA AO ITEM
}

EDA CASTRO LUCAS DE SOUZA

Doutora em Sociologia pelo Centro de Pesquisa e Pós-Graduação sobre as Américas da Universidade de Brasília (Ceppac/UnB).

Professora do Programa de Pós-Graduação em Administração da Universidade de Brasília (UnB). SQN, 1 16, Bloco I, ap. 208, Asa Norte, Brasília - DF - Brasil - CEP 70773-090

E-mail: edalucas@gmail.com

\section{GUMERSINDO SUEIRO LOPEZ JÚNIOR}

Mestre em Administração pelo Programa de Pós-Graduação em Administração da Universidade de Brasília (UnB). Administrador da Área de Planejamento do Banco Nacional de Desenvolvimento Econômico e Social (BNDES). Avenida República do Chile, 100, Centro, Rio de Janeiro - RJ - Brasil - CEP 20031-917 E-mail: gumersindojunior@uol.com.br

\section{ANTÔNIO CEZAR BORIIA}

Doutor em Engenharia pelo Departamento de Engenharia de Produção e Sistemas da Universidade Federal de Santa Catarina (UFSC).

Professor do Departamento de Engenharia de Produção e Sistemas da Universidade Federal de Santa Catarina (UFSC).

Caixa Postal 476, Campus Universitário, Trindade, Florianópolis - SC - Brasil - CEP 88010-970

E-mail: cezar@deps.ufsc.br

LUCIANO RICARDO RATH ALVES

Doutorando em Engenharia pelo Departamento de Engenharia de Produção e Sistemas da Universidade Federal de Santa Catarina (UFSC).

Caixa Postal 476, Campus Universitário, Trindade, Florianópolis - SC - Brasil - CEP 88010-970

E-mail: lucianorath@yaho.com.br

Este artigo pode ser copiado, distribuído, exibido, transmitido ou adaptado desde que citados, de forma clara e explícita, o nome da revista, a edição, o ano, e as páginas nas quais o artigo foi publicado originalmente, mas sem sugerir que a RAM endosse a reutilização do artigo. Esse termo de licenciamento deve ser explicitado para os casos de reutilização ou distribuição para terceiros. Não é permitido o uso para fins comerciais. 


\section{RESUMO}

A escala instrumento de medida de atitude empreendedora (Imae), desenvolvida por Souza e Lopes Jr. (2005), contém duas dimensões: prospecção e inovação, e gestão e persistência. Com a finalidade de verificar a validade e o intervalo em que propicia a medida de atitude empreendedora, além de investigar sua capacidade de discriminar a resposta que o indivíduo está apto a dar, o objetivo deste artigo é validar a escala Imae por meio do modelo de resposta gradual da teoria da resposta ao item (TRI), que revolucionou a teoria de medidas. A TRI, construtos da psicologia utilizados em estudos de discriminação de respostas, em especial em grandes amostras de respondentes a um determinado fenômeno, é constituída de modelos matemáticos que relacionam um ou mais traços latentes (não observados) de um indivíduo com a probabilidade de este dar uma determinada resposta a um item. O ponto crucial da TRI é que ela leva em consideração o item particularmente, sem relevar os escores totais, portanto as conclusões não dependem apenas do teste ou questionário, mas de cada elemento que o compõe. Os principais resultados encontrados foram a identificação de dois níveis da escala, denominados âncoras, que permitem interpretar tendências de pessoas com atitude empreendedora e a constatação de que os itens da escala Imae apresentam boa capacidade de discriminar a resposta que o indivíduo está apto a dar, o que confere qualidade aos itens e, portanto, à escala. A importância deste estudo reside no papel fundamental que a atitude desempenha nas escolhas que as pessoas fazem em relação à própria vida, de modo a ajudá-las a determinar seus próprios atos.

\section{PALAVRAS-CHAVE}

Atitude; Atitude empreendedora; Empreendedorismo; Teoria da resposta ao item; Medidas e métodos. 


\section{INTRODUÇÃO}

Uma escala para medir atitude empreendedora é importante porque a atitude desempenha um papel fundamental nas escolhas que os indivíduos fazem em relação à própria vida, de modo a ajudá-los a determinar os próprios atos (PETTY; WEGENER, I998). As atitudes constituem um complexo objeto de estudo da psicologia social, apresentando um grande número de definições. Guilford (I954) considera atitude como uma disposição pessoal comum aos indivíduos, que os impele a reagir a objetos, situações ou proposições em moldes que podem ser considerados favoráveis ou desfavoráveis. Lambert e Lambert (I972) dizem ser atitude uma maneira organizada e coerente de pensar, sentir e reagir em relação a pessoas, grupos, questões sociais ou, de modo geral, a qualquer acontecimento ocorrido no meio circulante. Rodrigues (I972) define o termo como o conjunto de crenças, sentimentos e tendências comportamentais dos sujeitos diante de um determinado objeto social.

Nessa linha, é possível dizer que o termo atitude está relacionado a avaliações favoráveis ou não, positivas ou não, feitas de diversas formas pelas pessoas em relação às outras, aos objetos e aos fatos. Essas avaliações estão baseadas em emoções, crenças, experiências passadas e comportamentos, sendo internamente consistentes ou ambivalentes.

Com base no entendimento de que atitudes incluem emoções, cognições e comportamento manifesto, podendo ser consideradas predisposições para avaliar objetos psicológicos (AJZEN, 200I), infere-se que, se a atitude for medida, é possível predizer qual será a conduta do indivíduo. Em função de atitudes, avaliam-se sentimentos, ações e escolhas que são, pois, elementos importantes na explicação do comportamento humano, principalmente por se constituirem em poderosos preditores desse comportamento.

Adjetivando atitude como empreendedora, é possivel considerá-la uma predisposição para o comportamento empreendedor, o qual envolve características do homem e do meio no qual se insere, representando, em sentido amplo, uma conduta voltada para o próprio homem, suas ações, visões de mundo e formas de transformar a realidade. Nessa lógica, quando se mensura a atitude das pessoas, pode-se explicar o comportamento empreendedor, ou seja, a decisão de criar negócio, de inovar e de agregar valor a empreendimentos, processos e produtos em organizações. A abordagem comportamental de empreendedorismo, no enfoque das atitudes como antecedentes do comportamento empreendedor, emerge na busca de suprir a fragilidade e as limitações de abordagens como as de traços de personalidades e características demográficas.

Com base na teoria do comportamento planejado de Ajzen (I99I) e no conceito de atitude empreendedora de Souza, Fracasso e Lopez Jr. (2008), foi 
criada por Souza e Lopez Jr. (2005) a escala instrumento de medida de atitude empreendedora (Imae), fundamentada, inicialmente, em quatro dimensões: inovação, realização, planejamento e poder. Com a finalidade de verificar a validade e o intervalo em que a escala Imae é propícia para mensurar a atitude empreendedora, bem como investigar sua capacidade de discriminar a resposta que o indivíduo está apto a dar, o objetivo deste artigo é validar essa escala por meio do modelo de resposta gradual da teoria da resposta ao item.

A teoria do comportamento planejado advoga que o comportamento é influenciado pela intenção de executá-lo, a qual depende de um juízo de valor fundamentado em uma das crenças que o indivíduo possui - a atitude. Atitude empreendedora, segundo Souza e Lopez Jr. (2005, p. 4), é definida como a "predisposição aprendida, ou não, para agir de forma inovadora, autônoma, planejada e criativa, estabelecendo redes sociais". Entre as alternativas da teoria da medida, utilizou-se a teoria da resposta ao item (TRI) para o tratamento estatístico, e o modelo adotado foi o de resposta gradual (MRG) dessa teoria.

\section{INSTRUMENTO DE MEDIDA DE ATITUDE EMPREENDEDORA (IMAE)}

Para identificar a presença da atitude empreendedora em empresários, foi realizada pesquisa, em 2004, com proprietários-gerentes de pequenas empresas varejistas e de serviço, participantes do Projeto Empreender do Distrito Federal, que apresentou como resultado a escala Imae.

Por meio de estudo realizado por Souza e Lopes Jr. (2005), o qual resultou em uma matriz de características do comportamento empreendedor, foram estabelecidas as quatro dimensões de análise utilizadas para a construção dessa escala:

- Planejamento: predisposição para gerenciar o empreendimento, a fim de obter informações para acompanhá-lo e avaliá-lo sistematicamente.

- Realização: predisposição para ter iniciativas, tomar decisões, atingir objetivos, cumprir metas, buscar oportunidades e aceitar riscos.

- Poder: predisposição para liderar, influenciar nas ações e nos resultados do empreendimento, estabelecer redes sociais e desenvolver a autoconfiança.

- Inovação: predisposição para agir de forma inovadora e criativa e construir diferenciais competitivos e produtivos.

A escala Imae (SOUZA; LOPES JR., 2005), composta de 36 itens, sendo I3 na dimensão planejamento, nove na dimensão realização, oito na dimensão 
poder e seis na dimensão inovação, utiliza a escala Likert com intervalo de I a Io, em que I significa "nunca apresenta atitude empreendedora" e Io indica "frequentemente possui essa atitude". Essa escala foi testada em uma amostra de 290 empresários. No resultado das análises das médias - desvios padrão, modas e frequências das variáveis - foi observado que proprietários-gerentes de pequenas empresas da região do Distrito Federal, de modo geral, possuíam as atitudes descritas nas questões do instrumento. Com a análise fatorial, verificou-se que as quatro dimensões - planejamento, realização, poder e inovação - foram agrupadas em dois fatores, os quais receberam novas denominações de acordo com as características dos itens que os constituíram: fator I (prospecção e inovação), com 20 itens e fator 2 (gestão e persistência), com 16 itens.

$\mathrm{Na}$ avaliação estatística exploratória, foi realizada análise fatorial, e verificaram-se, pelo exame dos componentes principais (ou Principal Components PC), um alto número de valores superiores a o,30, um índice Kaiser-Meyer-Olkin (KMO) de 0,90 e a presença de um ou dois componentes. As análises fatoriais dos eixos principais (ou Principal Axis Factoring - PAF) foram realizadas para os dois fatores positivos. $O$ fator prospecção e inovação apresentou um índice de consistência interna $\alpha=0,89$ e itens com cargas fatoriais variando entre 0,33 e $0,7 \mathrm{I}$, e o fator gestão e persistência apresentou um índice de consistência interna $\alpha=0,87$ e itens com cargas fatoriais variando entre o,3I e o,64 (SOUZA; FRACASSO; LOPEZ JR., 2008).

Embora a escala Imae tenha obtido bons índices no estudo exploratório, ressalta-se que ela continua em construção, passando pelo processo de análise confirmatória, com o propósito de ser revalidada em outros contextos com diferentes populações e culturas e ampliar sua aplicação e confiabilidade, na busca por maior compreensão da representação social do fenômeno empreendedorismo no Brasil (SOUZA; FRACASSO; LOPEZ JR., 2008).

\section{TEORIA DA RESPOSTA AO ITEM}

A TRI é formada por um conjunto de modelos matemáticos que visa relacionar a habilidade (traço latente) de um respondente e a probabilidade de esse indivíduo dar certa resposta a um item (questão) (ANDRADE; TAVARES; VALLE, 2000). O traço latente é uma variável não observada, enquanto a resposta é a variável - ou o estímulo - que pode ser vista (HAMBLETON; SWAMINATHAN; ROGERS, I99I).

Segundo Hambleton, Swaminathan e Rogers (I99I), a TRI baseia-se em dois postulados: 
- O desempenho do respondente pode ser predito ou explicado por um conjunto de habilidades.

- O relacionamento entre o desempenho do respondente no item e o conjunto de traços subjacentes pode ser descrito por uma função monotônica, crescente não linear, chamada função característica do item ou curva característica do item. Essa função indica que quanto maior for a habilidade do respondente, maior será a probabilidade de ele dar uma resposta afirmativa ao item.

Assim, para a TRI, a probabilidade de o indivíduo dar uma resposta correta depende da habilidade do respondente e das características do item.

Uma suposição necessária para que haja veracidade nos modelos de estimação dos parâmetros é que, para uma determinada habilidade, as respostas aos diferentes itens do teste sejam independentes; essa suposição é conhecida como independência local (ANDRADE; TAVARES; VALLE, 2000). Outra suposição comumente utilizada na TRI é a da unidimensionalidade, ou seja, a ideia de que o comportamento pode ser explicado por uma única habilidade dominante e de que os itens estão relacionados a essa única habilidade (HAMBLETON; SWAMINATHAN; ROGERS, I99I). Segundo Andrade, Tavares e Valle (2000), a unidimensionalidade implica independência local, portanto a primeira condição satisfaz as duas. Uma discussão detalhada sobre dimensionalidade aplicada à TRI pode ser vista em De Ayala e Hertzog (I99I).

De acordo com a necessidade de resolver problemas com diferente número de dimensões do traço latente e da natureza dos itens - com duas ou mais categorias, ordinais ou nominais -, foram criados variados modelos da TRI. O Quadro I apresenta modelos unidimensionais usuais.

\section{QUADRO I}

PRINCIPAIS MODELOS UNIDIMENSIONAIS DA TRI

\begin{tabular}{|c|c|c|}
\hline \multicolumn{2}{|c|}{ NATUREZA DO ITEM } & MODELO \\
\hline \multirow{3}{*}{ Original } & \multirow{3}{*}{ Dicotômico } & $\begin{array}{l}\text { Modelo logístico de } 1 \text { parâmetro ou Modelo de Rasch } \\
\text { (One-parameter logistic model ou Rasch model) }\end{array}$ \\
\hline & & $\begin{array}{l}\text { Modelo logístico de } 2 \text { parâmetros } \\
\text { (Two-parameters logistic model) }\end{array}$ \\
\hline & & $\begin{array}{l}\text { Modelo logístico de } 3 \text { parâmetros } \\
\text { (Three-parameters Logistic Model) }\end{array}$ \\
\hline
\end{tabular}

(continua) 
QUADRO I (CONCLUSÃO)

PRINCIPAIS MODELOS UNIDIMENSIONAIS DA TRI

\begin{tabular}{|c|c|c|}
\hline \multicolumn{2}{|c|}{ NATUREZA DO ITEM } & MODELO \\
\hline \multirow{5}{*}{ Original } & \multirow{9}{*}{ Politômico } & Modelo crédito parcial (Partial credit model) \\
\hline & & $\begin{array}{l}\text { Modelo crédito parcial generalizado } \\
\text { (Generalized partial credit model) }\end{array}$ \\
\hline & & Modelo de escala gradual (Rating scale model) \\
\hline & & Modelo de resposta gradual (Gradel response model) \\
\hline & & $\begin{array}{l}\text { Modelo Sequencial para respostas ordenadas } \\
\text { (Sequential model for ordered responses) }\end{array}$ \\
\hline \multirow{3}{*}{ Nominal } & & Modelo de resposta nominal (Nominal categories model) \\
\hline & & $\begin{array}{l}\text { Modelo de degraus para análise de crédito parcial } \\
\text { (Steps model to analyse partial credit) }\end{array}$ \\
\hline & & $\begin{array}{l}\text { Modelo de resposta para itens de múltipla escolha } \\
\text { (Response Model for mult-choice items) }\end{array}$ \\
\hline Desdobramento & & $\begin{array}{l}\text { Modelo de desdobramento gradual generalizado } \\
\text { (Generalized graded unfolding model) }\end{array}$ \\
\hline
\end{tabular}

Fontes: Andrade, Tavares e Valle (2000), Araujo, Andrade e Bortolotti (2009), Embreston e Reise (2000) e Van Der Linden e Hambleton (2007).

Andrade, Tavares e Valle (2000) informam que quanto mais categorias de resposta o item tiver, maior será a possibilidade de extrair informações. No entanto, o número de categorias é limitado pela amostra mínima e pelo número de respostas contidas em algumas das categorias, sob risco de não haver convergência dos algoritmos de estimação ou de haver erros demasiados. No que diz respeito ao tamanho mínimo da amostra, Edelin e Reeve (2007) afirmam não haver consenso entre os autores; por exemplo, enquanto alguns recomendam 200 respondentes para uma boa estimação dos modelos com mais de dois parâmetros, outros indicam 500.

A TRI permite posicionar sobre uma mesma escala o item e a habilidade do respondente, o que possibilita não apenas ordená-los, mas também fazer inferências qualitativas com respeito à sua posição na escala. Esse processo consiste em determinar conjuntos de itens (itens âncoras) pertencentes a determinados níveis da escala (níveis âncoras), dando condições para que os especialistas 
descrevam aspectos dos examinandos (BEATON; ALLEN, I992). Uma forma de determinar níveis e itens âncoras consiste em encontrar elementos que atendam a dois critérios (equações I e 2). Quando é encontrado pelo menos um item âncora, em determinado nível, este também passa a ser considerado âncora. Itens e níveis âncoras podem ser encontrados com as equações I e 2, nas quais Y é um nível discreto da escala imediatamente inferior a $\mathrm{Z}$, e $\mathrm{P}(\mathrm{U}=\mathrm{I} \mid \theta)$, uma função da probabilidade crescente de o respondente dar uma resposta afirmativa ao item, dada sua habilidade $\theta$ (BEATON; ALLEN, I992).

$$
\begin{aligned}
& P(U=\mathrm{I} \mid \theta=\mathrm{Z}) \geq 0,65 \\
& P(U=\mathrm{I} \mid \theta=\mathrm{Y})<0,5
\end{aligned}
$$

Na escala da TRI, a habilidade $\theta$ de um sujeito pode ter como medida qualquer múltiplo de desvio padrão dentro do intervalo $[-\infty ; \infty]$. É necessário, então, estabelecer um valor central para esse domínio. Normalmente, a métrica utilizada tem média $\mu=0$ e desvio padrão $\sigma=\mathrm{I}$. No entanto, para facilitar a interpretação, essa métrica pode ser alterada para $\mu^{*}$ e $\sigma^{*}$ sem perda na relação de ordem preexistente. Nas equações 3, 4 e 5, a* e b* são os parâmetros, e $\theta *$, a habilidade reparametrizada (ANDRADE; TAVARES; VALLE, 2000).

$$
\begin{gathered}
\mathrm{a} *=\frac{\mathrm{a}}{\sigma} \\
\mathrm{b} *=(\sigma \times b)+\mu) \\
P(U=\mathrm{I} \mid \theta *)=P(U=\mathrm{I} \mid \theta)
\end{gathered}
$$

\subsection{MODELO DE RESPOSTA GRADUAL}

O modelo de resposta gradual (MRG) proposto por Samejima (I969) é adequado para casos unidimensionais que tenham itens com mais de duas categorias de respostas ordenadas, como as de uma escala Likert. Segundo Samejima (2008, p. 563), a expansão da TRI para modelos politômicos "resultou em melhoria substancial da aplicabilidade da TRI para diversas áreas das ciências sociais e naturais". Entretanto, essa teoria tem sido aplicada não apenas nessas ciências, mas também em diversas áreas do conhecimento (BORTOLOTTI, 20IO).

No modelo genérico de resposta gradual, a probabilidade de o respondente escolher uma determinada categoria de resposta é dada pelas equações 6, 7 e 8 (SAMEJIMA, I969): 


$$
\begin{gathered}
P_{i, k}\left(\theta_{j}\right)=P_{i, k}^{+}\left(\theta_{j}\right)-P_{i, k+\mathrm{I}}^{+}\left(\theta_{j}\right) \\
P_{i, o}^{*}\left(\theta_{j}\right)=\mathrm{I} \\
P_{i, m+\mathrm{I}}^{+}\left(\theta_{j}\right)=0
\end{gathered}
$$

Em que:

- i é o item;

- j é o respondente;

- $\quad \mathrm{k}=\mathrm{O}, \mathrm{I}, 2 \ldots, \mathrm{m}_{\mathrm{i}}$, a k-ésima categoria de resposta e $\mathrm{m}+\mathrm{I}$, o número de categorias;

- $\quad \theta_{j}$ é a habilidade do respondente, que, nesse caso, representa a atitude empreendedora;

- $\quad \mathrm{P}_{\mathrm{i}, \mathrm{k}}\left(\theta_{\mathrm{j}}\right)$ é a probabilidade de o respondente com habilidade $\theta$ escolher a categoria $\mathrm{k}$, denominada função característica de operação (SAMEJIMA, 2008).

- $\quad \mathrm{P}_{\mathrm{i}, \mathrm{k}}^{+}\left(\theta_{\mathrm{j}}\right)$ é a probabilidade crescente em função da habilidade de o respondente escolher a categoria $\mathrm{k}$ ou mais alta. É denominada função característica de operação acumulada (SAMEJIMA, 2008). Para $P_{i, k}^{+}\left(\theta_{j}\right)$, Samejima (2008) propõe a utilização da função logística de dois parâmetros da TRI (Equação 9) (ANDRADE; TAVARES; VALLE, 2000):

$$
P_{i, k}^{+}\left(\theta_{j}\right)=\frac{\mathrm{I}}{\mathrm{I}+\mathrm{e}^{-\mathrm{a}_{\mathrm{i}}\left(\theta_{\mathrm{j}}-\mathrm{b}_{\mathrm{ik}}\right)}}
$$

Na qual:

- $\quad a_{\mathrm{i}}$ é parâmetro de discriminação do item;

- $\quad b_{i k}$ é o parâmetro de dificuldade da categoria $k$ do item i;

- $\quad \mathrm{P}_{\mathrm{i}, \mathrm{k}}^{+}\left(\theta_{\mathrm{j}}\right)$ é a probabilidade de o indivíduo selecionar uma categoria maior ou igual a k, em função de sua habilidade $\theta_{\mathrm{i}}$.

Quando se aplica a Equação 9 nas funções acumuladas ( $\mathrm{P}^{+}$...) das equações 6, 7 e 8, obtêm-se as funções características de operação para cada categoria do MRG (Equação Io):

$$
P_{i, k}\left(\theta_{j}\right)=\frac{\mathrm{I}}{\mathrm{I}+e^{-a_{i}\left(\theta_{j}-b_{i, k}\right)}}-\frac{\mathrm{I}}{\mathrm{I}+e^{-a_{i}\left(\theta_{j}-b_{i, k+1}\right)}}
$$

Nota-se, na Equação 9, que existem um parâmetro de discriminação por item e um parâmetro de dificuldade "b" por item e categoria, tal que:

$$
\mathrm{b}_{\mathrm{i}, \mathrm{I}}<\mathrm{b}_{\mathrm{i}, 2}<\ldots<\mathrm{b}_{\mathrm{i}, \mathrm{mi}}
$$


Os parâmetros dos itens e o traço latente devem ser estimados com métodos matemáticos, que utilizam algoritmos numéricos iterativos. Para estimação dos parâmetros dos itens, é usual utilizar o método da máxima verossimilhança marginal, e, para estimação dos traços latentes, os mais usuais são o método da máxima verossimilhança e os métodos bayesianos média a posteriori (expected a posteriori - EAP) e moda a posteriori (maximum a posteriori - MAP).

O detalhamento de tais modelos aplicados ao MRG pode ser visto em Samejima (I969). Nesse modelo, cada categoria do item fornece uma quantidade de informação variável em função de $\theta$ dada pela Equação II (SAMEJIMA, 2008). Na Equação II, $P_{i, k}{ }^{\prime}(\theta)$ e $P_{i, k}{ }^{\prime \prime}(\theta)$ são a derivada primeira e segunda de $P_{i, k}(\theta)$.

$$
\mathrm{I}_{\mathrm{i}, \mathrm{k}}(\theta)=\left(\frac{P_{i, k}^{\prime}(\theta)}{P_{i, k}(\theta)}\right)^{2}-\left(\frac{P_{i, k}^{\prime \prime}(\theta)}{P_{i, k}(\theta)}\right)
$$

A Equação I2 é a função de informação do item, em que $\mathrm{P}_{\mathrm{i}, \mathrm{k}}{ }^{(}(\theta)$ é a derivada de $P_{i, k}(\theta)$ (SAMEJIMA, 2008):

$$
\mathrm{I}_{\mathrm{i}}(\theta)=\sum_{\mathrm{i}, \mathrm{k}} \mathrm{I}_{\mathrm{i}, \mathrm{k}}(\theta) \times P_{i, k}(\theta) \equiv \sum_{i, k}\left(P_{i, k}^{\prime}(\theta)\right)^{2}
$$

E a Equação I3 é a função de informação do teste (medida):

$$
\mathrm{I}(\theta)=\sum \mathrm{I}_{\mathrm{i}}(\theta)
$$

Na escala da TRI, o erro padrão da medida também é uma função de $\theta$. No modelo genérico da TRI, o erro padrão EP é dado pela Equação I4 (ANDRADE; TAVARES; VALLE, 2000):

$$
\mathrm{EP}(\theta)=\frac{\mathrm{I}}{\sqrt{\mathrm{I}(\theta)}}
$$

\section{VALIDAÇÃO DA ESCALA IMAE}

Para analisar a capacidade de discriminar a resposta que o indivíduo está apto a responder e a qualidade aos itens da escala Imae, utilizaram-se a TRI e o MRG. Uma vez que a TRI apresenta problemas de estimação quando há poucas respostas em alguma categoria, as respostas originais foram recategorizadas, conforme a Tabela I. 
TABELA I

CATEGORIAS DE RESPOSTAS

\begin{tabular}{lcc}
\hline MRG & ESCALA ORIGINAL & \% DE RESPOSTAS \\
\hline 1 - Nunca & {$[0 ; 6]$} & 23 \\
\hline 2 - Raramente & 7 & 11 \\
\hline 3 - Às vezes & 8 & 17 \\
\hline 4 - Frequentemente & 9 & 33 \\
\hline 5 - Com muita frequência & 10 & 16 \\
\hline
\end{tabular}

Fonte: Elaborada pelos autores.

As respostas da pesquisa de Souza e Lopez Jr. (2005) foram submetidas ao processo de estimação dos parâmetros com o software Parscale ${ }^{\circledR}$ versão 4.I.2328.4 de 2003, creditado a Eiji Muraki e R. Darrell Bock. Nessa etapa, verificou-se, por meio do parâmetro de discriminação, a capacidade de os itens distinguirem a probabilidade de o respondente escolher uma das categorias com que se identifica. Quando há itens com pouca discriminação, estes são descartados, e um novo processo de estimação é efetuado com os remanescentes. Foram considerados pouco discriminantes itens com a $<0,70$, valor pouco superior ao da classificação proposta por Baker (200I), o qual considera haver baixa discriminação quando a < 0,65.

O segundo procedimento foi a verificação da curva de informação e do erro padrão da medida. Com isso, foi possível verificar a acuracidade do intrumento, a validade (PASQUALI, 2003) e o intervalo em que a escala é propícia para mensurar a atitude empreendedora.

A escala foi reparametrizada para simplificar a interpretação. Por fim, foram determinados itens e níveis âncoras para inferir aspectos da atitude empreendedora de indivíduos ou grupos em relação à sua posição na escala.

\section{CAPACIDADE DE ESCOLHA DE RESPOSTAS EM FUNÇÃO DO NÍVEL DE ATITUDE EMPREENDEDORA}

O parâmetro de discriminação médio do conjunto de itens ficou em I,34 com desvio padrão de o,3I. Pelo critério de discriminação mínimo sugerido por Baker (200I), de 0,65, e de Hambleton e Swaminathan (I985), de I,O, o teste propicia boa capacidade de os respondentes escolherem uma categoria de resposta em 
função do nível de atitude empreendedora. Nenhum item precisou ser descartado porque a discriminação mínima de o,8I encontrada, ocorrida nos itens 4 e I4, é maior que o valor convencionado de 0,70; assim, o instrumento continuou com os seus 36 itens.

Na escala centrada na média $\mu=0$ e com desvio padrão $\sigma=\mathrm{I}$, o parâmetro de dificuldade do teste (média dos parâmetros de dificuldade dos itens) é - 0,28 , com desvio padrão igual a 0,58 . Na Tabela 2, constam os itens e seus parâmetros de discriminação (a) e de dificuldade médio das categorias $\left(\mathrm{b}_{\mathrm{m}}\right)$.

\section{TABELA 2}

ITENS DO INSTRUMENTO E SEUS PARAMETROS DE DISCRIMINAÇÃO E DIFICULDADE MÉDIA DAS CATEGORIAS

\begin{tabular}{lllll}
\hline$N^{\circ}$ & ITEM & A & BM \\
\hline 1 & $\begin{array}{l}\text { Implemento novas ideias com o objetivo de melhorar a qualidade do } \\
\text { meu negócio. }\end{array}$ & 1,62 & $-0,09$ \\
\hline 2 & Exploro novas oportunidades de negócio. & 1,11 & 0,19 \\
\hline 3 & Mudo de estratégia, se necessário, para alcançar uma meta. & 1,38 & $-0,11$ \\
\hline 4 & Assumo riscos para expandir o meu negócio. & 0,81 & 0,68 \\
\hline 5 & Defino metas de longo prazo, claras e específicas. & 1,02 & 0,82 \\
\hline 6 & $\begin{array}{l}\text { Adoto procedimentos para assegurar que o trabalho atenda a padrões } \\
\text { de qualidade previamente estipulados. }\end{array}$ & 1,39 & $-0,28$ \\
\hline 7 & Utilizo contatos pessoais para atingir meus objetivos. & 1,24 & $-0,48$ \\
\hline 8 & Responsabilizo-me pela conclusão dos trabalhos nos prazos estipulados. & 1,29 & $-1,03$ \\
\hline 9 & Busco informações sobre possíveis clientes. & 1,06 & $-0,23$ \\
\hline 10 & Confio na minha capacidade de superar desafios. & 1,22 & $-1,09$ \\
\hline 11 & Busco novas maneiras de realizar tarefas. & 1,44 & $-0,39$ \\
\hline 12 & Faço projeções claras para o futuro do meu negócio. & 1,47 & $-0,02$ \\
\hline 13 & Junto-me aos empregados nas tarefas para cumprir os prazos. & 0,98 & $-1,15$ \\
\hline 14 & Utilizo estratégias deliberadas para influenciar pessoas. & 1,44 & $-0,64$ \\
\hline 15 & Reviso continuamente objetivos de curto prazo. & 0,69 \\
\hline 16 & Busco informações sobre meu ramo de negócio em diferentes fontes. & 0,30 \\
\hline & & $1,80 n t i a j$ \\
\hline
\end{tabular}




\section{TABela 2 (CONClusão)}

ITENS DO INSTRUMENTO E SEUS PARÂMETROS DE DISCRIMINAÇÃO E DIFICULDADE MÉDIA DAS CATEGORIAS

\begin{tabular}{|c|c|c|c|}
\hline$N^{0}$ & ITEM & A & BM \\
\hline 17 & Faço sacrifícios pessoais para concluir tarefas. & 1,10 & $-1,49$ \\
\hline 18 & $\begin{array}{l}\text { Mantenho meus objetivos mesmo diante de resultados que não são } \\
\text { satisfatórios inicialmente. }\end{array}$ & 0,96 & 0,09 \\
\hline 19 & Consulto meus registros de controle antes de tomar decisões. & 1,47 & $-0,13$ \\
\hline 20 & Removo meus esforços para superar obstáculos. & 1,91 & $-0,58$ \\
\hline 21 & Busco novas soluções para atender às necessidades de clientes. & 1,91 & $-0,79$ \\
\hline 22 & $\begin{array}{l}\text { Planejo as atividades do meu negócio subdividindo tarefas de grande } \\
\text { porte em subtarefas. }\end{array}$ & 1,14 & 0,62 \\
\hline 23 & Procuro criar novos serviços. & 1,33 & $-0,23$ \\
\hline 24 & $\begin{array}{l}\text { Assumo a responsabilidade pela resolução de problemas que possam } \\
\text { prejudicar o desempenho de meu negócio. }\end{array}$ & 1,34 & $-0,74$ \\
\hline 25 & Considero-me o principal responsável pelo desempenho do meu negócio. & 0,88 & $-1,01$ \\
\hline 26 & Confio na minha competência como fonte de sucesso do meu negócio. & 1,47 & $-0,96$ \\
\hline 27 & Emprego esforços extras para a conclusão de tarefas programadas. & 1,52 & $-0,71$ \\
\hline 28 & Desenvolvo ideias novas para a solução de problemas. & 1,94 & $-0,53$ \\
\hline 29 & Defino continuamente objetivos de curto prazo. & 1,20 & 0,52 \\
\hline 30 & Assumo riscos com intuito de superar a concorrência. & 0,90 & 0,32 \\
\hline 31 & $\begin{array}{l}\text { Crio novas rotinas, objetivando a melhoria do desempenho do meu } \\
\text { negócio. }\end{array}$ & 1,46 & 0,03 \\
\hline 32 & Ajo antes de ser pressionado pelas circunstâncias. & 1,23 & 0,24 \\
\hline 33 & Costumo calcular o risco envolvido nos negócios que faço. & 1,48 & $-0,29$ \\
\hline 34 & Calculo os riscos de novos investimentos. & 1,58 & $-0,49$ \\
\hline 35 & Estimulo o espírito de equipe entre meus funcionários. & 1,77 & $-0,59$ \\
\hline 36 & $\begin{array}{l}\text { Estimulo a participação dos funcionários na busca pela solução de um } \\
\text { problema. }\end{array}$ & 1,75 & $-0,65$ \\
\hline
\end{tabular}

Fonte: Elaborada pelos autores. 
Os itens foram analisados com discriminações extremas. Os itens 4 e I4 apresentaram a menor capacidade de discriminação (0,8I) e o item 2I a maior (I,9I). Pode-se dizer que, no item 2I "Busco novas soluções para atender às necessidades de clientes", os respondentes têm menor dificuldade de identificação com uma das categorias da escala do que nos itens 4 e I4. Observa-se, no gráfico do item 4 (Gráfico I, à esquerda), que um indivíduo com atitude empreendedora em torno de 0,7 tem uma diferença de apenas $5 \%$ de probabilidade (aproximadamente) de escolher qualquer uma das cinco categorias, e que é praticamente impossível distinguir a diferença da probabilidade de alguém que tenha um nível de atitude entre $-2,0$ e 0,5 escolher a categoria 4 ou 5 , sendo este um item razoável em relação ao 2I. No item 2I, é nítido que, para cada intervalo do nível de atitude, prevalece a escolha de uma categoria. Entre os níveis - 0,5 e o, por exemplo, prevalece a probabilidade de escolha da categoria 4. Observa-se, também, que, para um determinado nível da escala, as probabilidades de escolha de categoria são, em geral, distintas. Por exemplo, para o nível -I, as probabilidades de escolha das categorias I, 2, 3, 4 e 5 estão em torno de $15 \%$, 35\%, 40\%, I3\% e 2\%, respectivamente. O item 2I pode ser considerado como tendo muito boa discriminação.

\section{GRÁFICO I}

\section{CURVAS CARACTERISTICAS DE OPERAÇÃO DOS ITENS 4 E 2 I DA ESCALA IMAE}
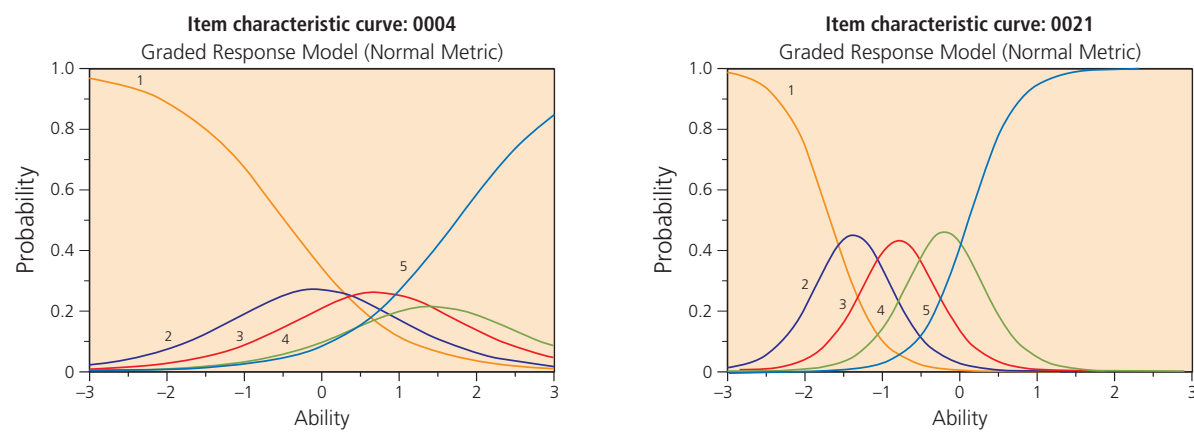

Fonte: Elaborado pelos autores.

O Gráfico 2 mostra dois itens que apresentam dificuldades diferentes, tendo discriminações semelhantes. Para o item com maior $b_{m}$ (item $5, b_{m}=0,82$, à esquerda no Gráfico 2), a probabilidade de uma pessoa escolher a categoria mais alta é superior às demais probabilidades somente em elevados níveis de atitude empreendedora (acima de I,3, na escala criada). Comparativamente, a probabilidade de uma pessoa escolher a categoria mais alta do item $\mathrm{I7}\left(\mathrm{b}_{\mathrm{m}}=-\mathrm{I}, 49\right.$, à direita no Gráfico 2) passa a ser a maior probabilidade já a partir de menores níveis de atitude empreendedora (nível -0,4 da escala). O mesmo ocorre para as probabilidades das 
demais categorias dos itens. Isso significa que a característica medida pelo item 5 - definição de metas de longo prazo, claras e específicas - ocorre somente em indivíduos com maiores níveis de atitude empreendedora. Já a característica medida pelo item I7 - realização de sacrifícios pessoais para concluir tarefas - pode ser observada em indivíduos com menores níveis de atitude empreendedora.

\section{GRÁFICO 2}

\section{CURVAS CARACTERÍSITICAS DE OPERAÇÃO DOS ITENS 5 E I7 DA ESCALA IMAE}
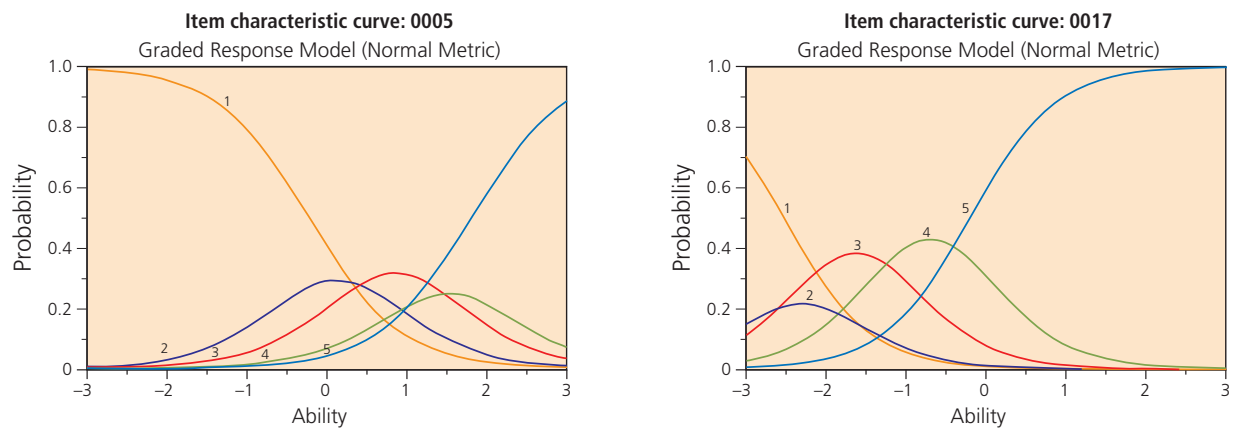

Fonte: Elaborado pelos autores.

Tomando como exemplo uma pessoa com nível de atitude empreendedora igual à média do grupo observado (nível zero na escala criada), observa-se que há maior probabilidade de a resposta ser "I: Nunca defino metas..." para o item 5 e "5: Com muita frequência, faço sacrifícios pessoais..." para o item I7. Pode-se concluir que um indivíduo com nível zero de atitude empreendedora apresenta a característica contida no item I7, mas não apresenta a característica medida pelo item 5 .

A escala apresentou alto nível de informação para mensurar pessoas que possuem atitude empreendedora mediana, com ápice em torno de $-0,6$, em que o erro padrão da medida é muito baixo. A escala Imae é propícia, também, para medir aqueles que se encontram num intervalo de atitude baixo a relativamante alto (-2; I) com pouco erro (Gráfico 3). Para expandir o intervalo adequado para medir indivíduos com alta atitude, podem ser incorporados ao instrumento itens definidos, pensando em características que somente devam ser observadas em pessoas com altos níveis de atitude empreendedora. O instrumento expandido pode ser aplicado a uma nova amostra, e os novos itens podem ser calibrados na mesma escala. Esse procedimento da TRI é denominado "equalização" e consiste em submeter alguns itens já calibrados juntamente com itens novos a uma nova amostra (ANDRADE; TAVARES; VALLE, 2000). 


\section{GRÁFICO 3}

\section{CURVA DE INFORMAÇ ÃO E ERRO PADR ÃO DA MEDIDA}

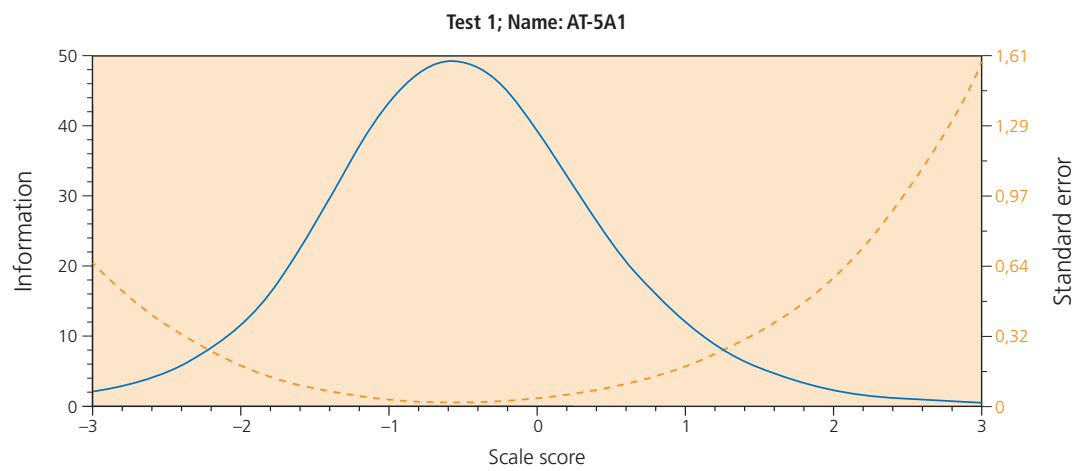

Fonte: Elaborado pelos autores.

A escala foi reparametrizada para média 30 e desvio padrão Io com o intuito de simplificar sua interpretação. Com esse procedimento, a escala passa a ter preponderantemente valores positivos. Aplicando as equações 3, 4 e 5, obtêm-se os valores reparametrizados expostos na Tabela 3.

\section{TABELA 3}

REPARAMETRIZAÇÃO DOS NÍVEIS DE ATITUDE EMPREENDORA

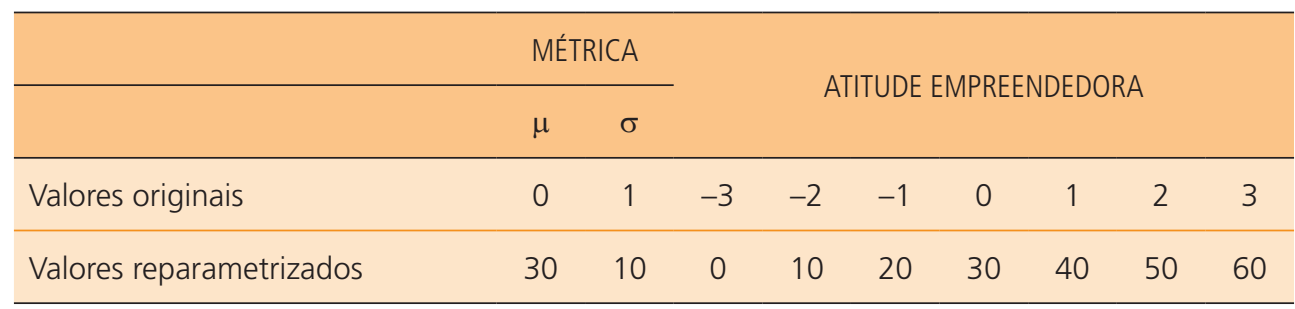

Fonte: Elaborada pelos autores.

A seguir, é exemplificada a determinação de itens e níveis âncoras reparametrizados, verificando se eles atendem às condições das equações I e 2 , considerando o nível médio de dificuldade dos itens. Na Tabela 4 , pode ser observado que o item I não atende à Equação 2 porque $\mathrm{P}(\mathrm{U}=\mathrm{I} \mid \mathrm{Y}=30)>0,50$, portanto ele não é um item âncora. Os itens 2I e 2 atendem às duas condições, sendo itens âncoras. O item 2I determina o nível âncora 30, enquanto o 2, o nível âncora 40. 
TABELA 4

EXEMPLO DE DETERMINAÇÃO DE ITENS E NÍVEIS ÂNCORAS

\begin{tabular}{|c|c|c|c|c|c|c|}
\hline \multirow{2}{*}{ ITEM } & \multirow{2}{*}{ PROBABILIDADE } & \multicolumn{3}{|c|}{ NÍVEL } & \multicolumn{2}{|c|}{ CONDIÇÃO } \\
\hline & & 20 & 30 & 40 & EQUAÇÃO 1 & EQUAÇÃO 2 \\
\hline 1 & & 0,19 & 0,54 & 0,86 & Sim & Não \\
\hline 21 & $\mathrm{P}(U=1 \mid \theta)$ & 0,40 & 0,82 & 0,97 & Sim & Sim \\
\hline 2 & & 0,21 & 0,45 & 0,71 & Sim & Sim \\
\hline
\end{tabular}

Fonte: Elaborada pelos autores.

No total, foram determinados I6 itens e 2 níveis âncoras (Quadro 2).

\section{QUADRO 2}

ITENS E NÍVEIS ÂNCORAS DA ESCALA IMAE

NIVEL
ÂNCORA ITEM

16 Busco informações sobre meu ramo de negócio em diferentes fontes.

20 Renovo meus esforços para superar obstáculos.

21 Busco novas soluções para atender às necessidades de clientes.

24 Assumo a responsabilidade pela resolução de problemas que possam prejudicar o desempenho do meu negócio.

3026 Confio na minha competência como fonte de sucesso do meu negócio.

27 Emprego esforços extras para a conclusão de tarefas programadas.

28 Desenvolvo ideias novas para a solução de problemas.

34 Calculo os riscos de novos investimentos.

35 Estimulo o espírito de equipe entre meus funcionários.

36 Estimulo a participação dos funcionários na busca pela solução de problemas.

(continua) 


\section{QUADRO 2 (CONCLUSÃO)}

\section{ITENS E NÍVEIS ÂNCORAS DA ESCALA IMAE}

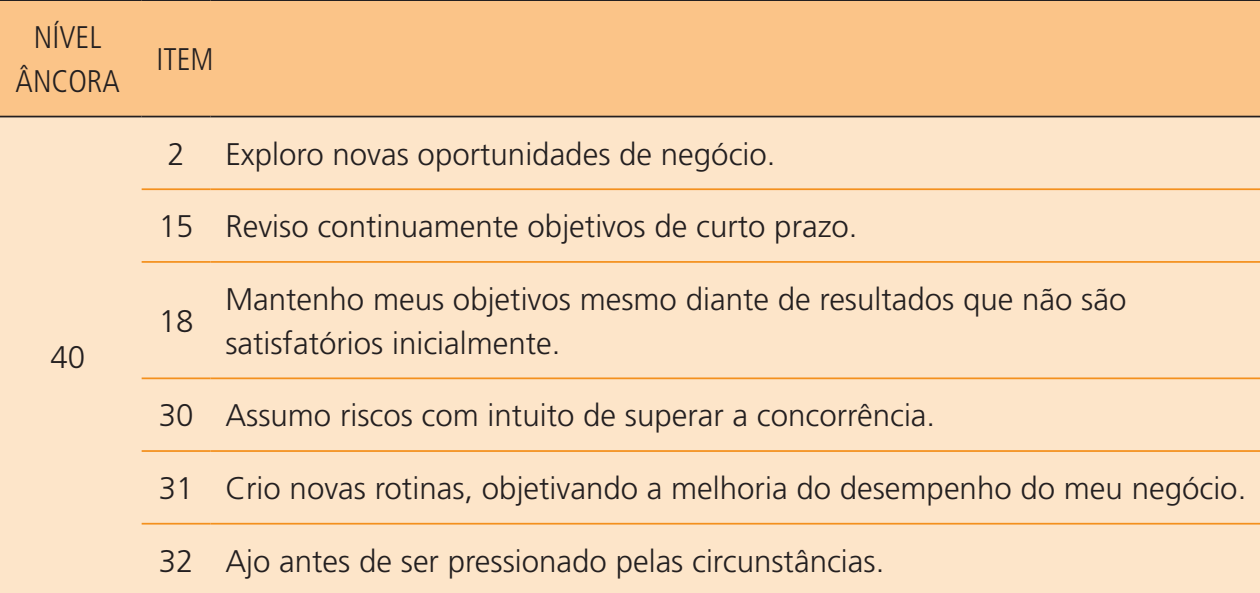

Fonte: Elaborado pelos autores.

Diante do processo de ancoragem, podem-se interpretar aspectos da atitude empreendedora de indivíduos que apresentem nível de atitude $\theta$ igual ou superior aos níveis âncoras. Os aspectos retratados são cumulativos; por exemplo, alguém que esteja posicionado no nível 40 ou superior da escala absorve, além dos aspectos do nível âncora 40, os do 30. Nas alíneas a seguir, estão as interpretações:

- Nível 30: busca informações sobre o ramo em que atua e calcula o risco de novos investimentos. Tem autoconfiança e tenacidade na condução do seu negócio. Assume a responsabilidade e procura engajar a equipe na resolução dos problemas. Procura utilizar a criatividade para melhor atender os clientes.

- Nível 40: é audacioso, procura investir em novos negócios. Tem maturidade e resistência às adversidades, principalmente nas primeiras fases do empreendimento. Enxerga possíveis infortúnios e age proativamente. Tem controle e flexibilidade para com os objetivos.

\section{CONCLUSÃO}

A escala Imae, após a validação por meio do MRG da TRI, continua com seus 36 itens, recategorizados em uma escala com cinco alternativas ordenadas de resposta cada (de I - nunca - a 5-com muita frequência). Os itens apresen- 
tam boa capacidade de discriminar a resposta que o indivíduo está apto a dar, o que confere qualidade a estes.

Constatou-se que existem itens com diferentes parâmetros de dificuldade para o respondente informar que toma determinada atitude com frequência, e esses itens identificam pessoas que possuem de baixa a alta atitude empreendedora. Para indivíduos com média atitude empreendedora, o instrumento proporciona elevado nível de informação e baixo erro padrão. Sob esse ponto de vista, a escala avalia bem os que se encontram nesse intervalo. O instrumento também estima bem aqueles indivíduos com baixa e moderadamente alta atitude empreendedora. Para medir alta atitude empreendedora com precisão, faz-se necessário criar e calibrar itens mais difíceis. Isso poderá ser feito sem que os dados coletados sejam descartados, ou ainda, sem que o intrumento seja descartado, porque a TRI tem como foco o item, e não o teste como um todo.

Além de poder posicionar pessoas ou grupos de pessoas na escala para fazer comparações, este estudo identificou dois níveis âncoras que permitem fazer análises qualitativas de aspectos da atitude empreenderora em função da sua posição na escala, apresentando tendências de os respondentes se classificarem nas dimensões constituivas da escala prospecção e inovação, e gestão e persistência. Ao reparametrizar a escala, prevaleceram valores positivos, o que permitiu interpretá-la como mais intuitiva.

A análise da escala Imae por meio da TRI/MRG identificou que a escala propicia boa capacidade de os respondentes escolherem uma categoria de resposta em função do nível de atitude empreendedora. Nessa linha, a referida escala constitui-se em uma tecnologia de gestão apropriada para medir atitude empreendedora, em especial de proprietários-gerentes de pequenas empresas.

\section{ENTREPRENEURSHIP ATTITUDE: VALIDATION OF A MEASUREMENT INSTRUMENT BASED ON GRADED RESPONSE MODEL OF THE ITEM RESPONSE THEORY}

\section{ABSTRACT}

The scale measurement instrument of entrepreneurship attitude (Imae), proposed by Souza and Lopes Jr. (2005), has two dimensions: prospection and innovation, management and persistence. Based on this scale it can be verified validity and interval that the entrepreneurship attitude may be measured, and also to verify its capacity of discriminating the answer that a human being is able to give. The purpose of this paper is to validate the Imae scale through the graded 
response model of the item response theory (IRT), which revolutionized the theory of measures. The IRT, psychology constructions used in discriminative answer studies, especially in large samples of respondents to a specific phenomenon, consist of mathematical models relating one or more latent traits (unobserved) of an individual with the probability of giving a particular response to an item. The main point of IRT is taking into account the particular item, without revealing the total scores, so the findings do not only depend on the test or questionnaire, but each item that composes it. Two levels of the scale were identified as the main results of this research. They are called anchors that allow interpreting people Entrepreneurship Attitude tendencies and findings that Imae items present good capacity in discriminating the answer that one is able to give, providing quality to those items, and, therefore, validating the Imae scale. This study is important in view of the role that attitude plays in the choices that individuals make about their own lives, helping to determine their own actions.

\section{KEYWORDS}

Attitude; Entrepreneurship attitude; Entrepreneurship; Item response theory; Measurements and methods.

\section{ACTITUD EMPRESARIAL: LA VALIDACIÓN DE UN INSTRUMENTO DE MEDIDA CON BASE EN EL MODELO DE RESPUESTA GRADUADA DE LA TEORÍA DE RESPUESTA AL ITEM}

\section{RESUMEN}

El instrumento de medición para la actitud emprendedora (Imae), escala desarrollada por Souza Jr. y Lopes (2005), contiene dos dimensiones: explotación y la innovación, y gestión y la persistencia. En base a esta escala, con el fin de verificar la validez y el intervalo que proporciona una medida de la actitud emprendedora, y investigar su capacidad para discriminar la respuesta que el individuo esta capaz de dar, el objetivo de este trabajo es de validar la escala Imae a través del modelo de respuesta graduada de la teoría de respuesta al item (TRI), que revolucionó la teoría de las medidas. La TRI, constructos de la psicología utilizados en los estudios de discriminación de las respuestas, especialmente en las grandes muestras de los encuestados en un fenómeno en particular, se compone de modelos matemáticos relacionados con uno o más rasgos latentes (no obser- 
vada) de un individuo con la probabilidad de dar una respuesta particular a un elemento. El punto principal de la TRI es que toma en cuenta el item en particular, sin relevar las puntuaciones totales, por lo que los resultados no sólo dependen de la prueba o cuestionario, sino de cada elemento que lo compone. Los principales resultados fueron la identificación de dos niveles de escala, llamados anclas, que permiten a interpretar las tendencias de las personas con actitud emprendedora y la comprensión de que los elementos de Imae tienen una buena capacidad para discriminar la respuesta que el individuo esta capaz de dar, lo que confiere la calidad de los elementos y por lo tanto de la escala. Este estudio es importante en vista del papel que juega la actitud en las elecciones que hacen los individuos sobre sus propias vidas, ayudando a determinar sus propias acciones.

\section{PALABRAS CLAVE}

Actitud; Actitud empreendedora; Empreendedor; Teoría de respuesta al item; Métodos y medidas.

\section{REFERÊNCIAS}

AJZEN, I. The theory of planed behavior. Organizational Behavior and Human Decision Process, n. 50, p. I79-2II, I99I.

AJZEN, I. Nature and operation of attitudes. Annual Review of Psychology, n. 52, p. 27-58, 200 I.

ANDRADE, D. F.; TAVARES, H. R.; VALLE, R. C. Teoria da resposta ao item: conceitos e aplicações. In: SIMPÓSIO NACIONAL DE PROBABILIDADE E ESTATÍSTICA (SINAPE), I4., 2000, Caxambu. Anais... Caxambu: Associação Brasileira de Estatística, 2000.

ARAUJO, E. A. C.; ANDRADE, D. F.; BORTOLOTTI, S. L. V. Teoria da resposta ao item. Revista da Escola de Enfermagem, v. 43, p. I000-1008, 2009.

BAKER, F. B. The basics of item response theory. 2. ed. Madison: ERIC Clearinghouse on Assessment and Evaluation, 200I.

BEATON, A. E.; ALLEN, N. L. Interpreting scales through scale anchoring. Journal of Education Statistics, v. I7, n. 2, p. I9I-204, I992.

BORTOLOTTI, S. L. V. Resistência à mudança organizacional: medida de avaliação por meio da teoria da resposta ao item. 20I0. 29I f. Tese (Doutorado em Engenharia de Produção)-Universidade Federal de Santa Catarina, Florianópolis, 20 o.

DE AYALA, R. F.; HERTZOG, B. A. The assessment of dimensionality for use in item response theory. Multivariate Behavior Search, v. 26, n. 4, p. 765-792, I99I.

EDELIN, M. O.; REEVE, B. B. Appling item response theory (IRT) modeling to questionnaire development, evaluation, and refinement. Quality of Life Research, v. I6, p. 5-18, 2007.

EMBRESTON, S.; REISE, S. P. Item response theory for psychologists. New Jersey: Lawrence Erlbaum Associates, 2000. 
FILION, L. J. O empreendedorismo como tema de estudos superiores. Empreendedorismo, ciência, técnica e arte. Brasília: CNI, IEL Nacional, 2000.

GUILFORD, J. P. Psychometric methods. 2. ed. New York: McGraw Hill Book Company, I954.

HAMBLETON, R. K.; SWAMINATHAN, H. Item response theory: principles and applications. Boston: Kluwer-Nijhoff, I985.

HAMBLETON R. K.; SWAMINATHAN, H.; ROGERS, H. J. Fundamentals of item response theory. Newbury Park: Sage, I99I.

LAMBERT, W. W.; LAMBERT, W. E. Psicologia social. Rio de Janeiro: Zahar, I972.

LOPES JR., G. S. Atitude empreendedora em proprietários-gerentes de pequenas empresas de varejo: criação de um instrumento de medida. 2004. 9I f. Dissertação (Mestrado em Administração)-Universidade Federal de Brasília, Brasília, 2004.

PASQUALI, L. Psicometria: teoria dos testes na psicologia e na educação. Petrópolis: Vozes, 2003. PETTY, R. E.; WEGENER, D. T. Attitude change: multiple roles for persuasion variables. In: GILBERT, D.; FISKE, S.; LINDZEY, G. (Ed.). The handbook of social psychology. 4. ed. New York: McGraw-Hill, I998. p. 323-389.

RODRIGUES, A. Psicologia social. 2. ed. Petrópolis: Vozes, 1972.

SAMEJIMA, F. Estimation of latent ability using a response pattern of graded scores. Richmond: Psychometric Society, I969. Disponível em: <http://www.psychometrika.org/journal/online/ MNi7.pdf>. Acesso em: i3 jul. 2010.

SAMEJIMA, F. Graded response model based on the logistic positive exponent family for dichotomous responses. Psichometrika, v. 73, n. 4, p. 56 I-578, 2008.

SOUZA, E. C. L. de; FRACASSO, E.; LOPEZ JR., G. S. Empreendedorismo e atitude empreendedora: conceitos e construção de escalas. In: ENCONTRO DE ESTUDOS SOBRE EMPREENDEDORISMO E GESTÃO DE PEQUENAS EMPRESAS, 5., 2008, São Paulo. Anais... São Paulo: Universidade Presbiteriana Mackenzie, 2008.

SOUZA, E. C. L; LOPES JR., G. S. Atitude empreendedora em proprietários-gerentes de pequenas empresas: construção de um instrumento de medida. Revista Eletrônica de Administração, v. II, n. 6, p. I-2I, nov./dez. 2005.

VAN DERLINDEN, W. J.; HAMBLETON, R. K. Handbook of modern item response theory. New York: Spring-Verlag, 2007. 